\title{
Assessment of In-Vehicle Messages in the Advance Warning Area of a Work Zone
}

\author{
Juan Li ${ }^{1}$, Qing $\mathrm{Li}^{1}$, Fengxiang Qiao ${ }^{1 *}$ and Lei $\mathbf{Y u}^{2,3}$ \\ ${ }^{1}$ Innovative Transportation Research Institute, Texas Southern University, Texas, USA \\ ${ }^{2}$ College of Science, Engineering, and Technology, Texas Southern University, Texas, USA \\ ${ }^{3}$ Xuchang University and Beijing Jiaotong University, China
}

\begin{abstract}
Simulations and field tests have shown that the connected vehicle technologies would not only enhance the mobility and safety of traffic operation, but also possibly reduce fuel consumption and exhaust emissions. So far, there is no consistent evaluation procedure on the perfomances of these in vehicle messages to drivers in work zone area. The objective of this research is to test, compare and develop a warning system to prevent occurring of work zone crashes and reduce vehicle emission. The main purpose of this study is to test and evaluate the different set of warning messages in the advance warning area of a work zone and develop an evaluation system for the warning system from the perspective of speed, acceleration rate, brake positions, lane changing positions, emission and work load. The work load of the three types of warning system is evaluated based on the NASA-TASK Load Index. The application of the mixed audio and image messages was able to reduce the overall vehicle emissions $\left(\mathrm{CO}_{2}, \mathrm{CO}, \mathrm{HC}\right.$, and $\mathrm{NO}_{\mathrm{x}}$ ). With the help of the both audio and image messages, drivers tend to recognize the road situation earlier than in the conventional situation and this will have a positively influence on driving safety. Especially, the mixed messages scenario outweights the other two scenarios in speed, acceleration rate, braking positions, emission, and work load.
\end{abstract}

Keywords: Driving simulator; In-vehicle warning message; NASA task load index; Evaluation system

\section{Introduction}

With the advancement of the new technologies in wireless communication, computer engineering, computer science, mechanical engineering, automatic control, the ground transportation system is very approaching its connected vehicle age with a lot of deployment studies and tests in implementation [1]. Simulations and field tests have shown that the connected vehicle technologies would not only enhance the mobility and safety of traffic operation, but also possibly reduce fuel consumption and exhaust emissions [2,3]. The research of connected vehicle technologies includes the modeling and simulation of the entire connected vehicle system [4], and the optimization of roadside unite (RSU) communication with vehicles [5].

The connected vehicle technologies allow providing instant roadside and/or in-vehicle messages, so as to properly guide drivers to drive even smoother for maximized benefits [3]. While there are a lot of reports on the successful applications of connected vehicle technologies in traffic operations such as the delima zone protection [6], signal priority requests [7], and work zone safety enhancement $[8,9]$, there is a lack of a systematic evaluation of effectiveness of the in-vehicle messages from the connected vehicle system.

According to Texas Motor Vehicle Crash Statistics 2016, there were 25,814 crashes occurring in work zones in 2016, with 162 fatal crashes and 181 fatalites in State of Texas [10]. The major causes of the crashes were drivers' fail to comply with the posted speed limit and the warning messages in work zone area [11]. Reports indicated that $10-35 \%$ of crashes in work zone happened in the advanced warning area [12].

Different warning systems could be used to convey information to drivers when they are driving through work zones. Some advocate the use of smartphone during driving, and found the smartphone-based messages could increase the drivers' awareness of roads sign in work zones [9]. Other ways to alert drivers include the inexpensive Bluetooth low-energy (BLE) tags [13] and smartwatch [14]. However, there is no consistent evaluation procedure on the perfomances of these in vehicle messages to drivers in work zone area.

\section{Research Objectives}

The objective of this research is to test, compare and develop a warning system to prevent occurring of work zone crashes and reduce vehicle emission. The main purpose of this study is to test and evaluate the different set of warning messages in the advance warning area of a work zone and develop an evaluation system for the warning system from the perspective of speed, acceleration rate, brake positions, lane changing positions, emission and work load. In addition, the work load of the three types of warning system is evaluated based on NASA-TASK Load Index.

\section{Methodology}

To measure the effectiveness of in-vehicle messages on drivers' driving behaviors, an evaluation matrix was proposed, which includes eight steps.

\section{Step 1}

Select candidate messages and test bed: Messages could serve for various purposes, such as navigation and warning for hazard, which could be varied with the test bed as well. In this study, in-vehicle warning messages that enhance the safety in a work zone are selected, which could be delivered with an audio or visual form.

\section{Step 2}

Design evaluation matrix: The effectiveness of the messages could be evaluated by drivers' driving performance measures, such as speeds and acceleration. Gernally, the evaluation should focus on safety, for example, whether drivers decelerate time to meet or maintain the posted speed. Besides, this study involves vehicle emissions and the workloads attributed to the messages.

${ }^{*}$ Corresponding author: Fengxiang Qiao, Innovative Transportation Research Institute, Texas Southern University, 3100 Cleburne Street, Houston, Texas, 77004 USA, Tel: 713-313-1915; Fax: 713-313-1856; E-mail: qiao_fg@tsu.edu

Received November 29, 2017; Accepted February 21, 2018; Published February 27,2018

Citation: Li J, Li Q, Qiao F, Yu L (2018) Assessment of In-Vehicle Messages in the Advance Warning Area of a Work Zone. J Civil Environ Eng 8: 302. doi: 10.4172/2165-784X.1000302

Copyright: @ $2018 \mathrm{Li} \mathrm{J}$, et al. This is an open-access article distributed under the terms of the Creative Commons Attribution License, which permits unrestricted use, distribution, and reproduction in any medium, provided the original author and source are credited. 


\section{Step 3}

Identify simulator or field test: Driving tests are required to exam the performance measures, which could be conducted either on a realroad, or in a lab environment. Though the field test is favorable to directly obtain drivers' real reponses to the test message, the open or closed road test environment is not fully controllable and it is difficult to gurantee the message is the main trigger for the responses. Further, the field tests are not awalys applicable to safety related driving tests, such as warning message for pedestrian crossing. Contrarily, in a lab test, all experimental environments are fully controllable, many kinds of crashes could be simulated, and more detailed driving behavior information could be recorded at a high sampling rate. Therefore, regarding safety warning message for a work zone area, a driving simulator test is preferable.

\section{Step 4}

Design test scenarios: Scenarios are designed in this step to achieve each objective of a study, which at least contains two scenarios, control and test scenario. For instance, to test the impacts of the in-vehicle messages on vehicle emissions, the scenario without warning message is required as a controlled one, and the scenario with warning messages is the test one.

\section{Step 5}

Recruit test subjects: Test subjects shall be recruited from the target group of the message application, namely drivers in United States (US), who hold a valid US drivers' license.

\section{Step 6}

Conduct the test and collect data: The driving test should be conducted by appointment. Subjects should be clearly informed the protocol and the requirement of the tests, and what and how data would collect from them. Before the tests, subjects should inform fully their consent. The specific methods are to collect data depend on the nature of the performance measures determined for the evaluation matrix. A driving simulator is able to collect drivers' performance measures, including driving speed, steer degree, braking level, and geolocation, up to a sampling rate of $60 \mathrm{~Hz}$.

\section{Step 7}

Processing and analyze drivers' driving behaviors: The collected data should be classified into their scenarios, which would be processed to estimate the change in drivers' peroformance measures from the base scenario.

\section{Step 8}

Weight and score the performance measures in the evaluation matrix: Each performance measure should be compared among scenarios. The favorable one is scored to 1 , otherwise, 0 . For example, if a test message in the scenario was more efficiently to enable subjects to lower their driving speed to meet the posted speed limit, the message is scored to 1 regarding safety, and otherwise, it is 0 . With repect to the concerns from various decision makers, a weight matrix is developed for each concern. For instance, when a full socre is 1 and the road safety is most concerned, the safety related performance measures, such as speed, acceleration, and lane change positions, shall be evenly or differentially distributed. The score for each concern would be summed up and compared among scenarios to identify the most effective messages.

\section{Developing an evaluation matrix}

An evaluation matrix is proposed to assess the impacts of the in- vehicle warning messages on safety, eco-driving, and mental work loads, which could be specifically measured by drivers' driving behaviors, vehicle emissions, and NASA-TASK Load Index.

\section{Safety performance measures}

Initially, in-vehicle warning messages are designed to improve drivers' driving behaviors to significantly reduce the possbile conflicts with ambient vehicles and traffic infrastructure, thereby enhancing safety. Strictly speaking, the compliance of the traffic control devices is the major performance measure, which could be reflected by drivers' driving behaviors. Therefore, in a work zone area, drivers' real-time driving speeds, acceleration rates, braking level, and lane change positions, are deemed as safty performance measures for the effectivenss of the in-vehicle warning messages.

\section{Eco-driving performance measure}

Vehicle emissions could be another concern for decision makers for better air quality. Though many studies demonstrated that the application of in-vehicle warning messages could redue vehicle emissions [13], the improvement could be varied with warning messages. The total emissions from each scenario serve as an eco-driving performance measure, which are predominantly estimated by the Motor Vehicle Emission Simutor (MOVES) approved by US Environmental Protection Agency (EPA). The major input variable is the operational mode ID distribution. The operational mode ID is classified by vehicle speed, acceleration, and vehicle specific power (VSP) [15].

VSP is the instantaneous tractive power demanded by aerodynamic drag, acceleration, rolling resistance, grade, per unit vehicle mass, which could be calculated using Equation (1)

$$
V S P=v^{*}[1.1 \times a+9.81 \times \operatorname{grade}(\%)+0.132]+0.000302 \times v^{\wedge} 3
$$

where ${ }^{v}$ is driving speed $(\mathrm{m} / \mathrm{s}),{ }^{a}$ is acceleration rate $\left(\mathrm{m} / \mathrm{s}^{2}\right)$, and grade (\%) is the vehicle vertical rise divided by slope length. In this study, the grade is zero.

\section{Mental workload performance measures}

In-vehicle warning messages could raise extra mental workload to drivers, which could be assessed by multidimensional subjective workload assessment instruments, such as NASA Task Load Index (NASA-TLX), Subjective Workload Assessment Technique (SWAT), and Workload Profile (WP).

Among all the workload assessment methods, NASA Task Load Index is a multi-dimensional scale to measure the one or more operators' workload when performing a task [16]. Compared with other workload assessment method, subjective rating may be the most appropriate way to measure the mental workload and can provide the most generally valid and sensitive indicator $[17,18]$. Thus NASA-TLX is selected to evaluate drivers' workload in this paper.

NASA-TLX consists of six subscales and each represents independent clusters of variables, namely mental demand, physical demand, temporal demand, frustration, effort, and performance. Mental demand defines how much mental and perceptual activity was required during driving test. This detriment is used to measure whether the scenario tested easy or demanding, simple or complex. Physical demand was used to describe how much physical activity was required during test. That is how much strength was involved in driving under different road sign warning scenarios. Temporal demand is used to measure how much time pressure the participant feel due to the rate or pace at which the driving task was executed. Performance is an indicator 
to measure the participants' personal evaluation of how successful they are in accomplishing the task. Effort is an index to measure how hard the participant works both mentally and physically during the test. The last index frustration level is used to measure the feeling that accompanies the participants during their execution of the task. The combination of these factors is used to measure the workload of the people who are performing a task.

NASA task load index uses a workload comparison card to evaluate the weight of each index. Participants are required to compare each pair in the 15 cards and circle the factor that outweighs the other. Then the result was tallied, and the corresponding weight was calculated.

When the participants finished the comparison cards, they are required to give a rating on the six factors on a rating sheet. They have to mark in the appropriate position to evaluate from low to high for the factors like mental demand, physical demand, temporal demand, effort and frustration, or from good to poor for the factor "performance". Then the raw rating results for each factor will be marked down. The Adjusted Rating is calculated by multiplying the weight and raw rating results.

\section{Driving simulator test}

Participants: A total of fifty participants, were recruited for the driving simulator test conducted in June, 2017. Among them, 31 are male and 19 are female, ranging in age from 19 to 36 . The average age of participants is 28 years old and a mean driving experience of 7 years. The criterion for selecting the participants was the possession of a driving license and at least 1 year of driving experience.

Equipment: This research is conducted using a driving simulator, DriveSafety DS-600c. The driving simulator could provide drivers with a fully immersive and integrated driving simulation scene with audio, visual, and dynamic effects realized. During the test, subjects sit in a real full-width automobile cab to drive in front of a $270^{\circ}$ wrap-around display screen. Meanwhile, the simulator monitors subjects' real-time driving behaviors, including speeds, braking levels, lane changes, and steer levels, at a sampling rate of $60 \mathrm{~Hz}$.

Test procedure and data collection: The driving simulator test was conducted by individual appointment. Subjects were instructed the test protocol and fully inform their consent before the driving test. There are three parts in the test, including training to operate a driving simulator, formal driving test, and NASA-TLX survey. The training is designed to allow subjects to be familiar with the driving simulator operation, and the simulated driving environment before the formal driving test. During the formal driving test, each subject was requested to drive through a work zone area like driving on the real road to experience no or pre-set in-vehicle warning messages for the work zone traffic control. After the driving test, the subject was immediately proceeded with NASA-TLX survey, which was instructed by test operator individually.

Scenario design: Three scenarios in the work zone simulation are designed: work zone with no audio or image warning system, work zone with a voice warning system, and work zone with a mixed warning system of audio and image. The specific timing that the image or voice warning messages for a work zone area is illustrated in Figure 1.

The configuration of the work zone in Figure 1 was designed according to the Manual on Uniform Traffic Control Devices (MUTCD). Each subject started from $973.7 \mathrm{~m}$ away the transition area of the work zone in the three scenarios and end at the $187 \mathrm{~m}$ after the traffic sign "Lane Ends Merge Left". When a vehicle drives into the designated areas, around $10 \mathrm{~m}$ close to a traditional traffic control device, the audio

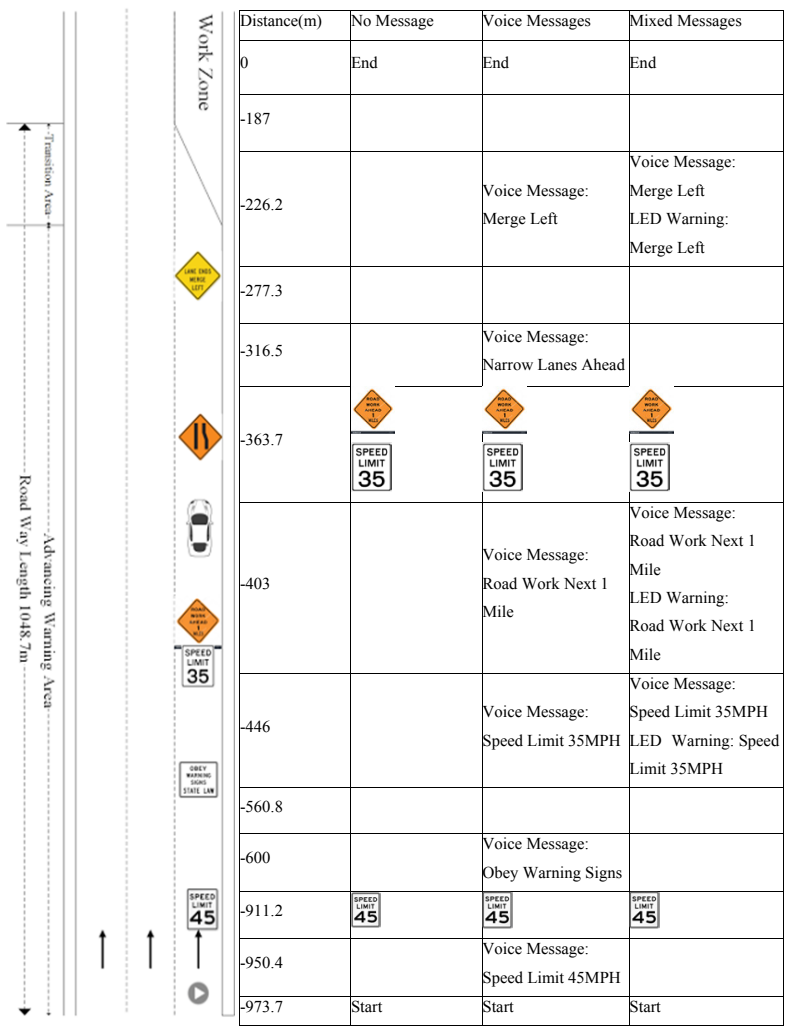

Figure 1: Message placements of all scenarios.

or image system will be actuated immediately. Basically, the in-vehicle warning messages in this study are consistent with the traditional traffic control device information, but in visual or audio forms. The message of the traffic sign is converted to audio or image instruction and delivered to the passing vehicle the same time the driver sees the corresponding traffic sign. The distance is calculated according to the minimum required reaction distance of $39.2 \mathrm{~m}$ (128.6 feet) when vehicles are moving at a speed of $56.32 \mathrm{~km} / \mathrm{h}(35 \mathrm{MPH})$. The distances are from the 2001 AASHTO Green Book and are for dry conditions. During the test, participants are requested to drive through a work zone on a suburban road.

\section{Results and Discussion}

\section{Speed profiles}

One of the ways to improve work zone safety is the appropriate use of speed limits to control vehicle speeds. Figure 2 is the speed profile of the three scenarios. In Figure 2a, the horizontal axis is the distance travelled during the test. The distance is calculated from the point “- 825 " because at that point the drivers tends to attain a comparatively constant velocity and this makes the three scenarios more comparable. The distance ends at "0" because at that point most of the participants stop when they enter the work zone. The velocity data was collected individually by the simulator and then interpolated according to a distance interval of 1 meter. Then the mean value of the interpolated results was calculated for each scenario.

As indicated in Figure $2 b$, the speed profile of the No Message scenario is indicated by green line on the top, and then comes the Mixed Messages scenario in blue and finally the Voice Messages scenario in red color at the bottom. In general, the speed profiles of the three scenarios 
are decreasing steadily as the messages constantly give the drivers messages to slow down and be prepared to lane changing. Without the warning of voice and image messages, participants in the No Message scenario tends to drive at a higher speed than the other two scenarios during the whole test route. The speeds of other two scenarios are lower than the No Message scenario.

In Figure 2, the road way was divided into 3 stages, stage 1 starts from the starting point to "- 492 ", in which drivers tend to attain a constant speed before beginning to decelerate. In this stage, the lines of Voice Messages scenario and Mixed Messages scenario interwine and this indicates the same speed level. Stage 2 is from the point "-492" to "-166". When the participants got the messages warning that there are work zone ahead and speed limit is $35 \mathrm{MPH}$, all the participants reduced their speed, with the speed line of No Messages scenario on the top and then comes the Mixed Message scenario and the voice message scenario on the bottom. Stage 3 starts from the point "- 166 " to " 0 ", in which drivers kept a relatively lower and constant speed after the road sign "Merge Left" or after hearing or seeing the corresponding voice or image messages until they stops. When entering Stage 3, the speed of the Mixed Messages was reduced to the lowest among the three scenarios, with No Messages still on the top.

Figure $2 b$ further illustrates the distribution of speed by percentage in the three stages. In the 9 subfigures, the $\mathrm{x}$ axis is the 9 speed bins that include all the velocity datas; the $\mathrm{Y}$ axis is the percentage of each bin data. The unit for speed is $\mathrm{km} / \mathrm{h}$.
At Stage 1, the speed limit is $72 \mathrm{~km} / \mathrm{h}$, most of the participants could comply with the speed limit. It should be noted that in the No Message scenario, there are still a relatively certain percentage of speed $\mathrm{s}$ are over the speed limit. Specially, in the Mixed Messages scenario, higher percentage (42\%) of speed falls with the scope of " $60-70$ " $\mathrm{km} / \mathrm{h}$, which shows the image and voice messages have great impact on the drivers' speed and as most of them are warned by the signs conveying the warning message of obeying signs.

In Stage 2, participants in the three scenarios got the message either by road sign or by voice or images that road work is 1 mile ahead and speed limit is $35 \mathrm{MPH}$, their speed are gradually reduced to below 70 $\mathrm{km} / \mathrm{h}$. However, there are still a relatively higher percentage of speed that are above $70 \mathrm{~km} / \mathrm{h}$ in the No Message scenario as compared with the other two scenarios.

In Stage 3, after two road signs conveying further information of "narrow lanes ahead" and "merge left", drivers were ready to enter the work zone. In this stage, most of the speeds are within the speed bin of " 50 to 60 " $\mathrm{km} / \mathrm{h}$, once again the Mixed Message scenario showed its low speediness priority that most the participants reduced their speed to below $60 \mathrm{~km} / \mathrm{h}$. In the No Message and Voice Messages scenarios, relatively higher percentages of speeds are in the range of above 60 $\mathrm{km} / \mathrm{h}$. A further deeper interpretation from these figures could be that the voice messages and image messages greatly affect the drivers' driving behavior of reducing speed and raised their awareness of obeying road signs.

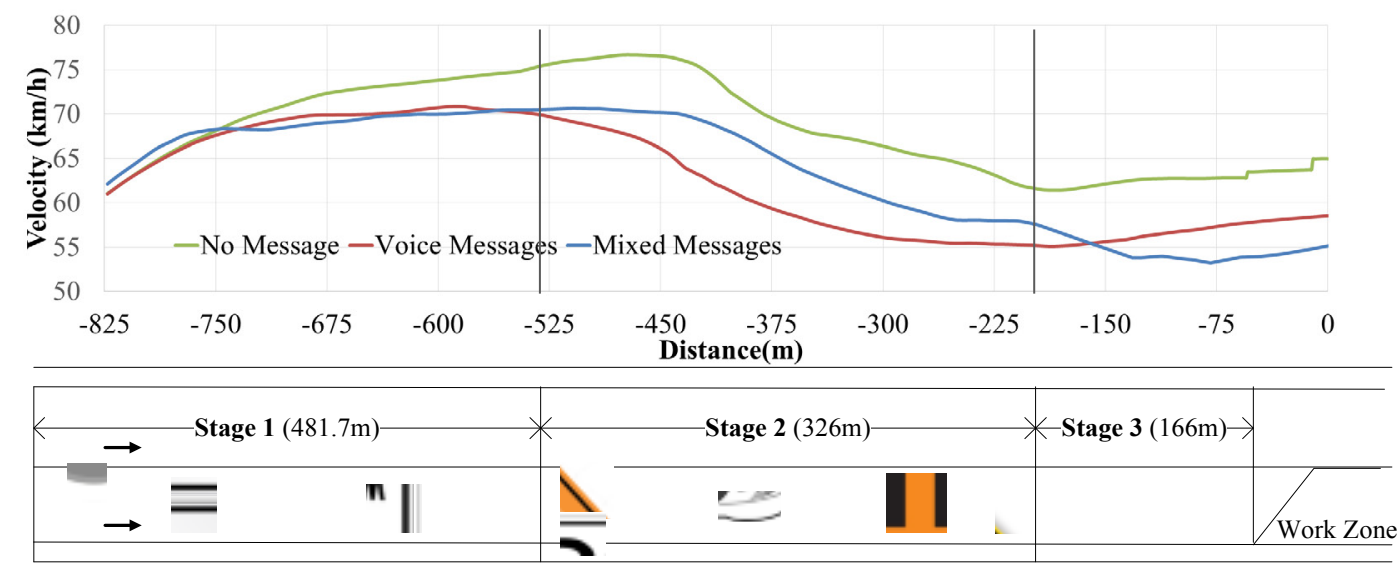

(2a): Speed profiles of the three scenarios.
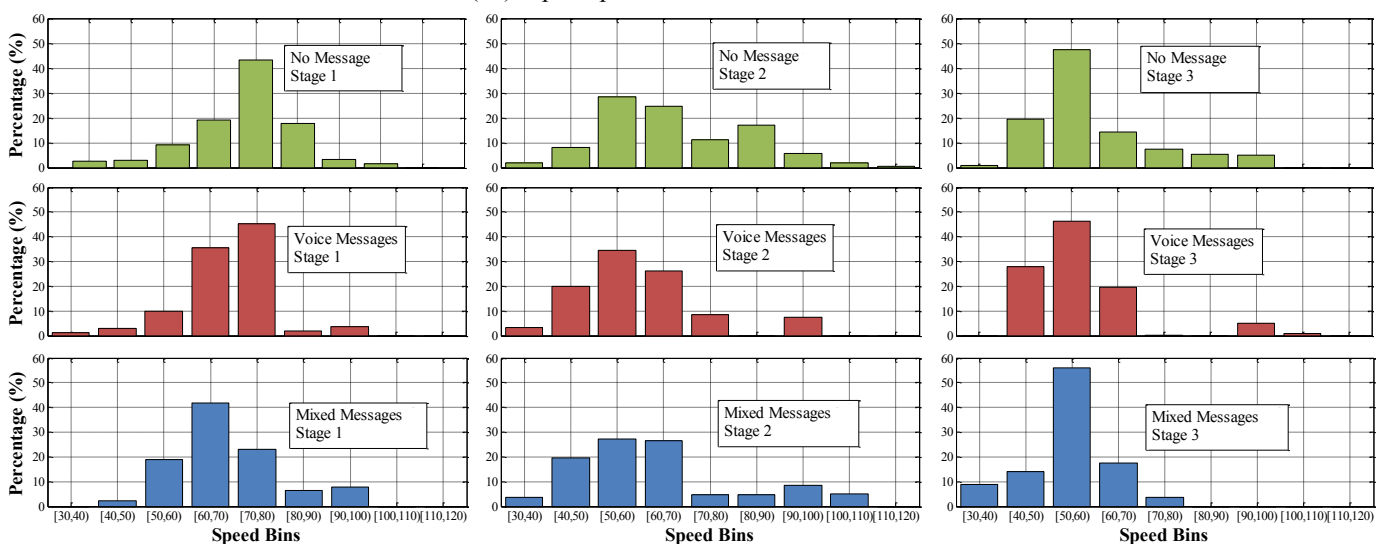

(2b) Speed bin profiles for different stages

Figure 2: Speed profiles of three scenarios. 


\section{Acceleration distributions}

Research shows that speed variance positively correlated with accident rates. Larger speed variance in work zones may lead to higher accident rates. Accident rates could be decreased by reducing the speed variance or having the vehicles travelled at the average speed. Figure 3 is the acceleration rate distributions of the three scenarios.

In the driving simulator DriveSafety DS-600c, the data was collected every $1 / 60$ second. The purpose of this analysis is to count and compare the acceleration rate in the three scenarios. In Figures $3 a-3 c$, the horizontal axis represents the acceleration range bins; the vertical axis is the percentage of the acceleration rate bins.
As the test ends when the participants enter the work zone, the acceleration data after point "0" was not counted in this study. As Figure 3 shows, most of the acceleration rates fall with the scope of "-1" to " 1 ". There are 98.12 percent, 98.6 percent and 99.68 in the range of "-1" to " 1 " in the three scenarios respectively. According to AASHTO, the comfortable deceleration rate is greater than $-3.4 \mathrm{~m} / \mathrm{s}^{2}$. According to Verizon (2018), for small and light vehicles, hard brake is " -3.92 " $\mathrm{m} / \mathrm{s}^{2}$. These two criterions are adopted as a dividing point to set the bin range and are indicated by the two dotted lines in the figures correspondingly.

As indicated in Figure 3a and Figure 3b, there do exist some percentage of deceleration rates that are below "-3.4" in the No

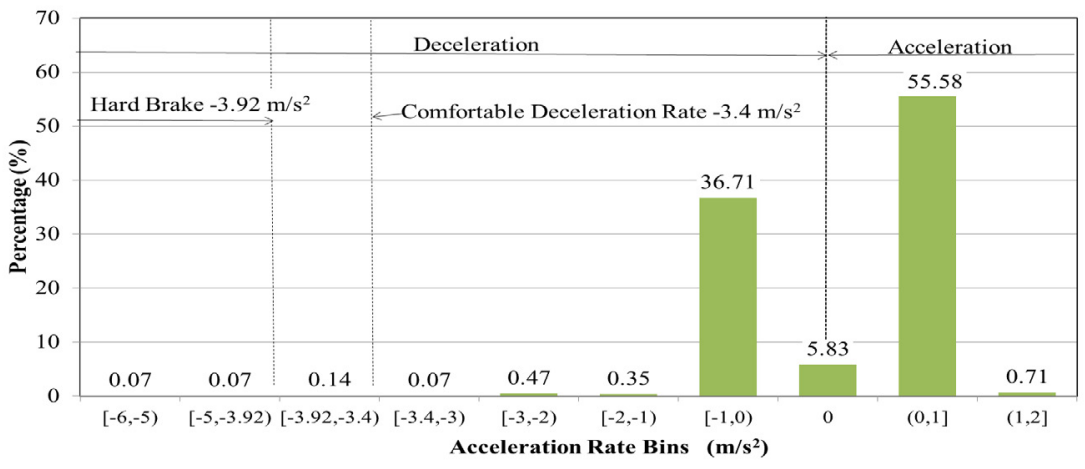

3a: Acceleration rate bins in the No Message scenario

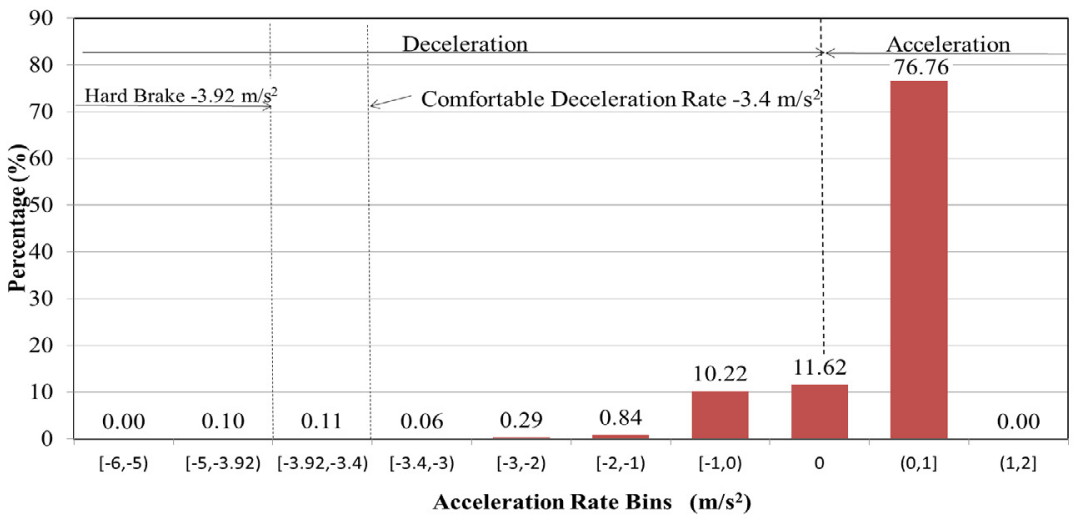

3b: Acceleration rate bins in the VoiceMessages scenario.

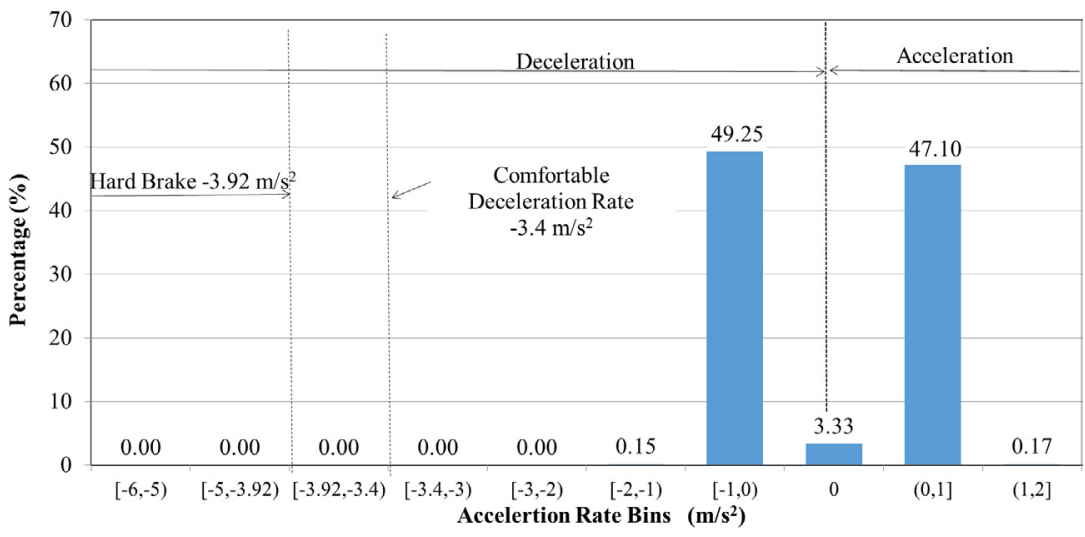

3c: Acceleration rate bins in the Mixed Messages scenario

Figure 3: Distribution of the acceleration rate in three scenarios. 
Message scenario and the Voice Messages scenario. This shows that some participants are beyond the comfortable deceleration rate. Some deceleration rates are even greater than the hard brake rate in the first two scenarios. Especially in the No Message scenario, 0.14 percent of acceleration rate is below the hard brake criterion of " -3.92 " $\mathrm{m} / \mathrm{s}^{2}$, and 0.28 percent is below "- 3.4 ". By contrast, in the Mixed Messages scenario, almost all the acceleration rate lies within the scope of "- 1 " to " 1 ", there is no deceleration rate that are beyond the comfortable deceleration rate and hard brake.

These results could be explained as the image messages may have a better warning effect for the drivers when they are driving during the test and timely image or voice warning could make drivers make less deep deceleration specially, the acceleration rate of " 0 " is chosen as a criterion because travelling at average speed means less traffic accidents. As illustrated in the three figures, 5.83 percent of are driving at average speed in the No Message scenario, 11.62 percent drivers Voice Messages scenario and 3.33 percent in the Mixed Messages scenario. From this perspective, the voice message may lead to less change in speed during the test.

\section{Braking positions}

Timely braking positions mean more safety in the work zone area. The braking positions of each participant was marked and indicated in the Figure 4 for the three scenarios. In the figures, the horizontal axis is the distance and the $\mathrm{Y}$ axis is the extent the driving press on the brakes. In Figure 4a, the five lines as marked by square, triangular, cross, asterisk and circle stand for the five road sign location in the test, the accurate position was marked by the textbox on the top. As indicated in the figure, most of the drivers brake down when they started because they saw the road sign of " $45 \mathrm{MPH}$ ". Then a comparative larger majority of participants brakes when they saw the road sign of "Road Work Next 1 Mile and Speed Limit 35 MPH" at the position of “-364".

Figure $4 \mathrm{~b}$ shows the braking positing in the Voice Messages Scenario. As indicated in the figure, most of the drivers first brake before they sight the first road sign message and the corresponding voice message was heard. Other drivers brake when the road sign "Road Work Next 1 Mile and Speed Limit $35 \mathrm{MPH}$ " and the corresponding voice message.

Quite different from the previous two scenarios, it is interesting to see that in the Mixed Message scenario, the majority of drivers brake after the road sign "Merge Left" and the corresponding voice message and image message. It could be explained that in this scenario, the image of "Merge Left" and the corresponding voice warning is conspicuous and the drivers change lane at the last moment before entering the work zone.

\section{Lane changing positions}

Earlier lane changing ahead of the work zone area implicates more safety to the drivers. Figure 5 is the distribution of the lane changing positions in the three scenarios. The lane changing positions data was collected by observing the moving track of each participant. The simulator kept record of the subject $\mathrm{X}$ and subject $\mathrm{Y}$ information of each participant and this could be reflected in a coordinate. When the driver takes the first action to merge from the existing lane, the point of distance was marked down by the Simulator.

As the first warning sign and voice message "Obey Warning Sign" start at the position " -600 ", lane changing action made before " -625 " was not calculated. The lane changing positions which were made after "0" point were also not calculated because at that point the driver had entered the transition area of the work zone. Then the normal distribution value was calculated according to the mean value and standard deviation.

As illustrated in Figure 5, the scenario No Message has the lowest vertex, which shows drivers tend to change their lanes at decentralized positions. By contrast, the distribution of the other two scenarios tends to be more concentrated, the reason being that the voice and image messages intervene drivers' decision of lane changing. To take a close look at the two controlled scenarios, this phenomenon is enhanced in the Mixed Messages scenario, which has the highest vertex as shown in the blue line. Participants in the mixed message scenario tend to change lanes at comparatively concentrated positions. Furthermore, the lane changing positions in the mix messages scenario are more ahead of the other two scenarios, as illustrated by the axises of symmetry which intersects the vertex. The fact that the first voice and image message that conveys the key information of work zone "Road Work Next 1 Mile, Speed Limit $35 \mathrm{MPH}$ " in the Mixed Message scenario are given at the point of " -446 " may explain this phenomenon. Participants in this scenario are more obeyed when they heard the voice message and saw the image ahead of their vehicles. The participants in the other two scenarios changed lanes at later points after the voice message "Narrow Lanes ahead" was given at "-316" and road sign "Narrow Lanes Ahead" at “-277”.

From Figure 5 it can be concluded that the Voice Messages scenario and Mixed Messages scenarios have better control of the drivers' lane changing behavior, especially when there are no other vehicles on the road, the earlier action of changing lanes means safer and secured pass of the work zone.

\section{Total emissions}

Possible vehicle emission factors of the three scenarios are estimated and shown in Figure 6, which includes four emission indexes: $\mathrm{CO}_{2}, \mathrm{CO}$, $\mathrm{HC}$, and $\mathrm{NO}_{\mathrm{x}}$.

In Figures 6a-6d, notable differences were found that the Voice Messages scenario has the highest emission factor in the four indexes wheras the Mixed Messages scenario has the lowest emission factor. Specially, the emission factor in the Mixed Messages scenario is 0.3 $\mathrm{g}$ less in the $\mathrm{CO}_{2} 12 \mathrm{mg}$ less in $\mathrm{CO}, 0.2 \mathrm{mg}$ less in $\mathrm{HC}$ and $1.7 \mathrm{mg}$ less in $\mathrm{NO}_{\mathrm{x}}$ and $0.12 \mathrm{mg}$ less than the Voice Messages scenario. The scenario No Message is in between the scenario Voice Messages and the scenario Mixed Messages.

\section{Drivers' Workload}

After the driving test, participants were required to evaluate the workload from six perspectives, mental demand, physical demand, temporal demand, performance, effort and frustration. The comparasion sequences of the six factors include:

- Effort/performance

- Temporal demand/frustration

- Temporal demand/effort

- Physical demand/frustration

- Performance/frustration

- Physical demand/ temporal demand

- Physical demand/performance

- Temporal demand/mental demand 


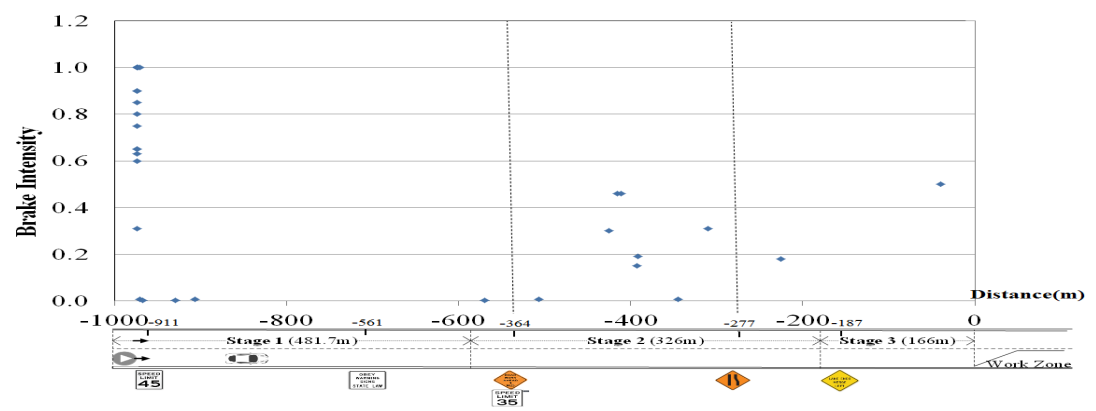

4a: Braking positions of no message scenario

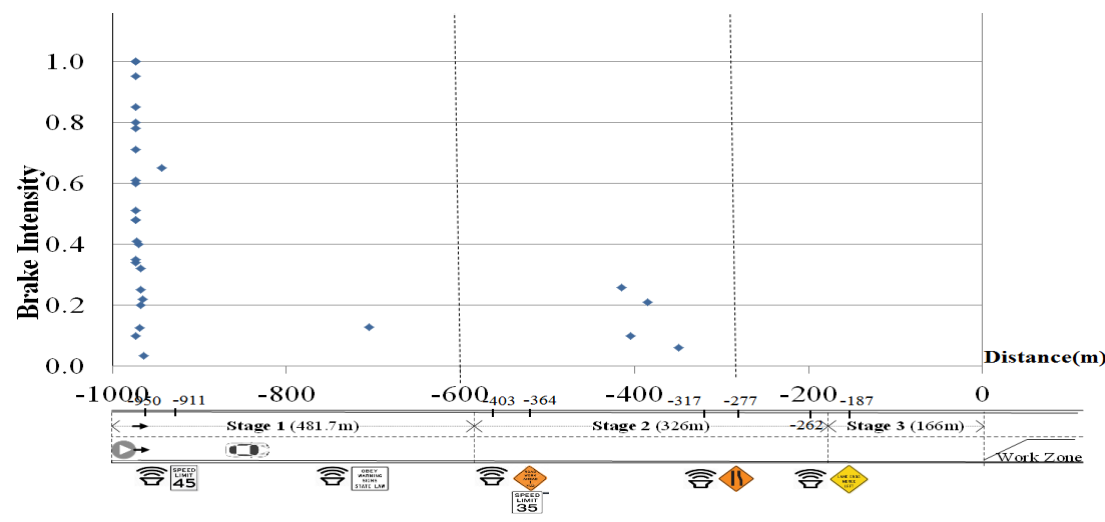

$4 \mathrm{~b}$ : Braking positions of voice messages scenario

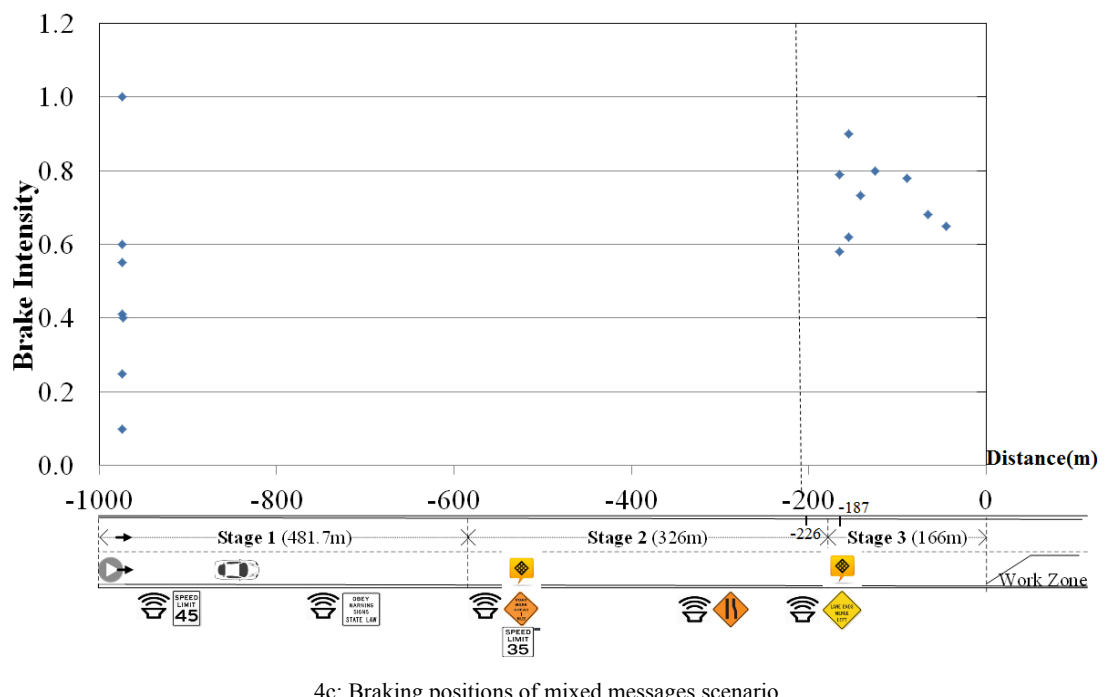

Figure 4: Braking positions of the three scenarios.

- Frustration/effort

- Performance/mental demand

- Performance/temporal demand

- Mental demand/effort

- Mental demand/physical demand

- Effort/physical demand

- Frustration/mental demand
Figure 7 shows the weights of the six factors for NASA-TLX Test for the 3 Scenarios. As illustrated in Figure 7, all the participants in the three scenarios put the highest weight on mental demand and lowest rate on physical demand. The results show that obeying traffic warning signals and messages during driving requires most mental efforts and drivers are much concentrated in minds. Therefore, drivers are not physically tired during this process. In the three scenarios, Temporal Demand has the second highest weight, which shows that driving through a work zone while obeying warning signs put much time pressure on the drivers, and the decision making of braking, lane changing, accelerating and decelerating require temporary and quick 


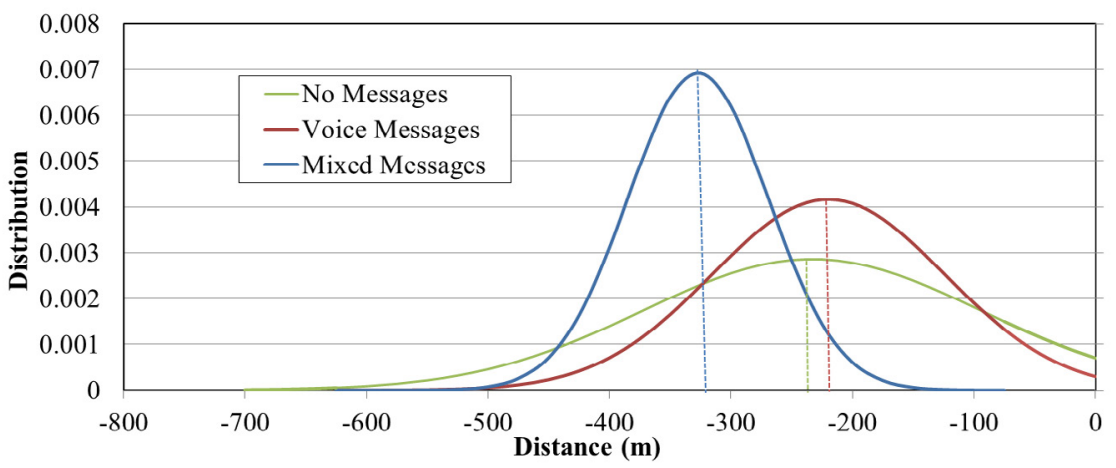

Figure 5: Distribution of lane changing positions in three scenarios.
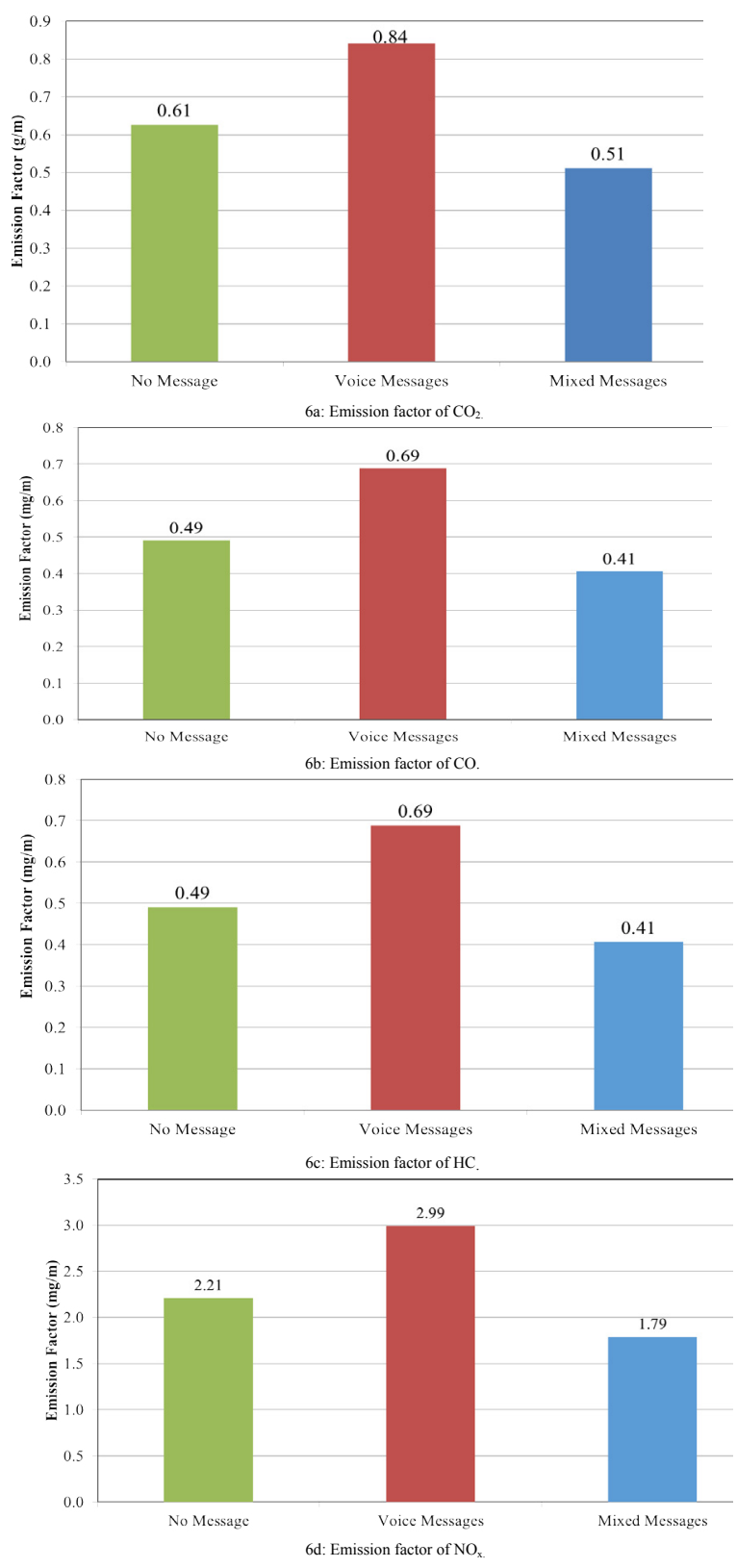

Figure 6: Emission factors of the three scenarios.

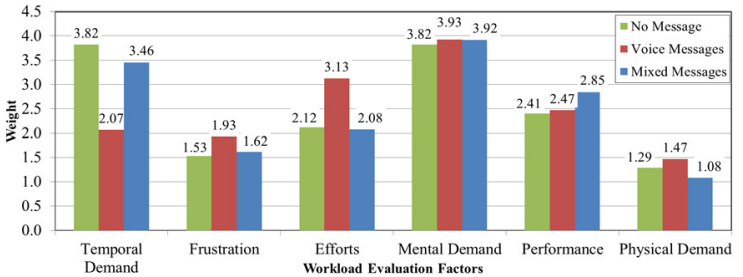

Figure 7: Weights of the Six Factors for NASA-TLX Test for the three scenarios.

response. Meanwhile, the weight for the Voice Message scenario has comparatively lower weight than the other two scenarios. In contrast, the Voice Message scenario has comparatively higher weight than the other two in the factor "Efforts". Besides Physical Demand, the low weight of Frustration shows that participants feel relatively relaxed and complacent during the completement of the task.

Table 1 is the mean weighted workload score for each scenario. From Table 1 it can be learned that the No Message scenario got a total of 11,730 and mean value of 690 workload score. The Voice Messages scenario got a total of 9008 and mean value of 600 workload score. The Mixed Messages scenario which contains mixed sign in its test has a total of 8375 workload value and an average of 582 . This is contray to the author's expection because voice message tends to have more workload during drivng because the driver has to obey warning message twice the number than the No Message scenario. A proper explanation could be the voice and image message provide a comapartively relexed environment that the driver do not need to constantly pay attention to the road sign and they could drive ahead when there is no sound our image ahead of them. This also could explain the fact that some people like to drive with their GPS voice message on even if they know the route, because the voice messge give them no work load and even make them relaxed and sure that they are driving in the right route.

\section{Evaluation system}

Based on the above discussion, an evaluation Matrix was established to evaluate the performaces of the three scenarios. Three aspects, as indicated by six factors, speed, acceleration rate, lane changing positions, braking positions, emission and work load are evaluated in this paper. Table 2 lists the factors tested in this paper and their weight and score. The original score was assigned according to the results analysed in this paper. Assume equal weight was attached to the six factors, that is $1 / 6$ for each factor, the No Message Scenario has 0 score, the Voice Messages gets 1/6, and the Mixed Messages gets 5/6. If safety 


\begin{tabular}{|c|c|c|c|c|c|c|c|c|}
\hline Scenario & Mental Demand & Physical Demand & Temporal Demand & Performance & Effort & Frustration & Total & Average \\
\hline No Message & 3,608 & 906 & 2,224 & 2,166 & 1,901 & 925 & 11,730 & 690 \\
\hline Voice Messages & 1,585 & 953 & 2,344 & 1,938 & 1,495 & 693 & 9,008 & 600 \\
\hline Mixed Messages & 2,765 & 890 & 1,200 & 1,955 & 1,510 & 415 & 8,375 & 582 \\
\hline
\end{tabular}

Table 1: Mean weighted workload score.

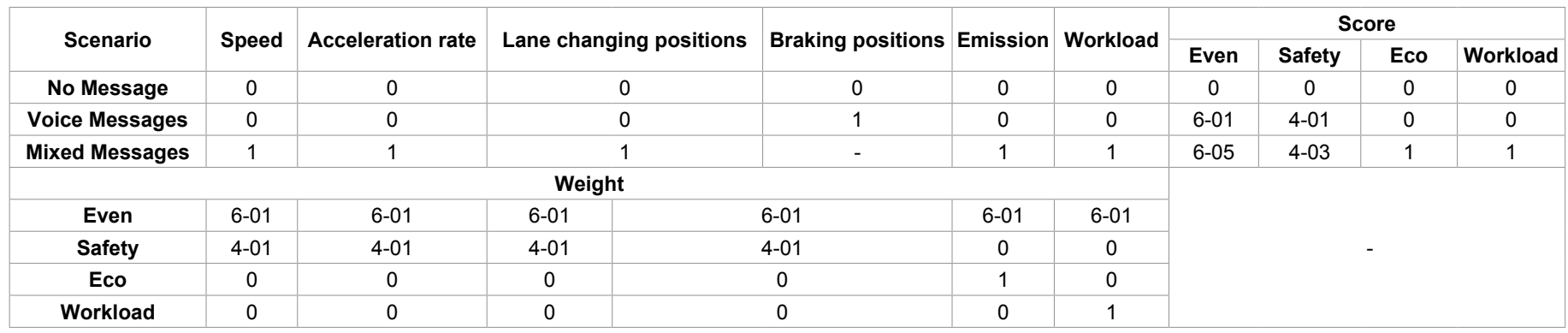

Table 2: Evaluation matrix.

is considered and $1 / 4$ is assigned to the factors speed, acceleration rate, lane changing positions, braking positions. Then $0,1 / 4$, and $3 / 4$ will be the score for the three scenarios respectively. If eco factors are considered, 1 will be attached exclusively to the emission factor and then the Mixed Messages scenario will have 1 in this case. Likewise, if work load is considered as the sole factor to evaluate the warning systems, the Mixed Messages scenario will have the full mark of 1 wheras the other two scenarios will have 0 .

\section{Conclusion}

In this paper, an evaluation system was established to evaluate the three-work zone warning message systems and thus to enhance driving safety and improve air quality. The impacts of the three systems on driving behaviors were tested by simulated driving scenarios. Three types of warning messages (no message, voice messges and mixed messages) were designed to compare the warning effects in terms of speed, acceleration rate, braking positions, and lane changing positions, emission factors and work load. NASA Work Load Index was employed to evaluate the drivers' work load of the warning messages while driving.

Results show that there are significant differences between the Mixed Messages scenario and the other two scenarios. With the help of the both audio and image messages, drivers tend to recognize the road situation earlier than in the conventional situation and this will have a positively influence on driving safety. Especially, the Mixed Messages scenario has better performance than the other two scenarios in speed, acceleration rate, braking positions, emission, and work load. Participants in the Mixed Messages scenarios drive at comparatable lower speed in the advanced warning area of the work zone. They were driving more steadily and do not have deceleration rates that are beyond the comfortable range. Meanwhile, the lane changing positions in the Mixed Messages scenario are also more ahead of the other two scenarios, which could put the driver at a safer condition before entering the work zone. Regarding the emission factor, the application of the mixed messages (audio and image) was able to reduce the overall vehicle emission in $\mathrm{CO}_{2}, \mathrm{CO}, \mathrm{HC}$ and NOx. This could contribute to a healthier environment in the modern city. This result indicates that the mixed warning messages could enhance the transportation safety in work zone.

The Voice Messages scenario surpasses the other two scenarios in terms of lane changing positions. Meanwhile, the voice warning messeges system has the highest emission factor.
In the furture study, more participants should be recruited to conduct simulated test about the results of different warning messages. Participants with wider demographic representation should be invited to enlarge the sample representation. If possible, large scale road test should be conducted to validate the results of the warning systems.

\section{Acknowledgements}

The authors acknowledge that this research is supported in part by the National Science Foundation (NSF) under grants \#1137732. The opinions findings, and conclusions or recommendations expressed in this material are those of the author(s) and do not necessarily reflect the views of the funding agencies.

\section{References}

1. Gopalakrishna D, Garcia V, Ragan A, English T, Zumpf S, et al. (2016) Connected Vehicle pilot deployment program phase 1, Human Use Approval Summary-ICF/Wyoming. United States. Dept. of Transportation. ITS Joint Program Office.

2. Olia A, Abdelgawad H, Abdulhai B, Razavi SN (2016) Assessing the potential impacts of connected vehicles: Mobility, Environmental, and Safety perspectives. J Intell Transportation Sys 20: 229-243.

3. Zulkefli MAM, Mukherjee P, Sun Z, Zheng J, Liu HX, et al. (2017) Hardware-inthe-loop testbed for evaluating connected vehicle applications. Transp Res Part C Emerg Technol 78: 50-62.

4. Songchitruksa P, Sunkari S, Ugalde I, Rosca J, Aparicio OJ (2017) Interlinking Vissim and ns-3 for connected vehicle simulation: Case study of intelligent dilemma zone avoidance. Transportation research record: J Transportation Res Board. pp. 36-43.

5. Olia A, Abdelgawad H, Abdulhai B, Razavi S (2017) Optimizing the number and locations of freeway roadside equipment units for travel time estimation in a connected vehicle environment. J Intell Transportation Sys 21: 296-309.

6. Zha L, Zhang Y, Songchitruksa P, Middleton DR (2016) An integrated dilemma zone protection system using connected vehicle technology. IEEE Trans Intell Transp Syst 17: 1714-1723

7. Gende M, Chowdhury M, Dey KC, Sarasua W (2016) Connected vehicle technology for allowing priority requests at signalized intersections-An analysis. Transportation Research Board $95^{\text {th }}$ Annual Meeting.

8. Abdulsattar $\mathrm{H}$, Mostafizi $\mathrm{A}$, Wang $\mathrm{H}$ (2017) Assessing the impacts of connectedvehicle technology on work zone rear-end collisions: Agent-based modeling approach (No. 17-01645). Transportation Research Board 96 th Annual Meeting Washington DC, United States.

9. Qiao F, Rahman R, Li Q, Yu L (2016) Identifying smartphone based intelligent messages for worker's crossing in work zones. J Transportation Technol 6: 76-85.

10. Texas Department of Transportation (2016) Texas motor vehicle crash statistics. TXDOT.

11. Weng J, Xue S, Yan X (2016) Modeling vehicle merging behavior in work zone merging areas during the merging implementation period. IEEE Trans Intell Transp Syst 17: 917-925. 
Citation: Li J, Li Q, Qiao F, Yu L (2018) Assessment of In-Vehicle Messages in the Advance Warning Area of a Work Zone. J Civil Environ Eng 8: 302. doi: 10.4172/2165-784X.1000302

Page 10 of 10

12. Qiao F, Rahman R, Li Q, Yu L (2017) Safe and environment-friendly forward collision warning messages in the advance warning area of a construction zone. Int J Intell Transportation Sys Res 15: 166-179.

13. Haseman R, Wasson J, Bullock D (2010) Real-time measurement of travel time delay in work zones and evaluation metrics using bluetooth probe tracking. Transportation Research Record: J Transportation Res Board 2169: 40-53.

14. Samost A, Perlman D, Domel AG, Reimer B, Mehler B, et al. (2015) Comparing the relative impact of smartwatch and smartphone use while driving on workload, attention, and driving performance. Proceedings of the Human Factors and Ergonomics Society Annual Meeting 59: 1602-1606.
15. Jimenez-Palacios JL (1998) Understanding and quantifying motor vehicle emissions with vehicle specific power and TILDAS remote sensing. Massachusetts Institute of Technology, Cambridge, UK.

16. Hoonakker P, Carayon P, Gurses AP, Brown R., Khunlertkit A, et al. (2011) Measuring workload of ICU nurses with a questionnaire survey: The NASA Task Load Index (TLX). IIE Trans Healthc Syst Eng 1: 131-143.

17. Qiao F, Zhang R, Yu L (2011) Using NASA-task load index to assess drivers' workload on freeway guide sign structures. In ICCTP: Towards Sustainable Transportation Systems 4342-4353.

18. Verizon (2018) Harsh braking and acceleration - Why monitor? 